\title{
MAMMOS Readout with Magnetic Field Modulation
}

\author{
N. Takagi, K. Mitani, H. Awano", K. Shimazaki", and N. Ohta* \\ Hypermedia Research Center, Sanyo Electric Co., Ltd., 180, Ohmori, Anpachi-cho, Anpachi-gun, Gifu 503-0195, Japan \\ *Tsukuba Research Laboratory, Hitachi Maxell Ltd., 6-20-1, Kinunodai, Yawara-mura, Tsukuba-gun, Ibaraki 300-2496, Japan
}

\begin{abstract}
MAMMOS (Magnetic AMplifying Magneto-Optical System) is one of the highest density readout methods for storage media. The signal is directly amplified on the medium and its amplitude is just the same as that from a long domain. Even at a $0.04 \mu \mathrm{m}$ domain, the signal was large enough. At a $0.2 \mu \mathrm{m}$ packed domain, $\mathrm{CNR}$ was more than $50 \mathrm{~dB}$ and BER was in the $10^{-4}$ range. By employing a MAMMOS readout with magnetic field modulation (MFM-MAMMOS), the signals have both very high resolution and large amplitude characteristics.
\end{abstract}

Key words: magneto-optical disk, magnetic amplifying, MAMMOS, MFM, high density recording, error rate

\section{Introduction}

The recording density of optical disks is generally limited by the diameter of the optical stylus for read/write. The improvement of the recording density has therefore mainly depended on the development of shorter wavelength lasers. On the other hand, in a magneto-optical disk it is possible to obtain signals from smaller domains under the optical limitation by using a Magnetically induced Super Resolution (MSR) disk [1][2].

The Advanced Storage Magneto-Optical disk (AS-MO) has a capacity of 6 GBytes in a 120-mm-sized single side disk due to the adaptation of MSR and magnetic field modulation (MFM) as the read/write strategy [3][4]. The capacity is larger than that of a 4.7-Gbyte DVD-ROM. Using MFM recording, magneto-optical disks have more advantages in the formation of smaller domains than phase change disks. There are reports of the recorded domain being as small as $1 / 10$ of laser beams especially in laser pumped MFM [5][6]. As in magnetic recording, readout techniques are the most important issue for high-density recording. As mentioned above, AS-MO has emerged as a solution of MSR.

MSR makes an aperture in the readout layer using the temperature distribution of the laser beam. Since the signals are reproduced through the aperture just like an isolated domain, we obtain the large signal without degradation of the optical transfer function (OTF). However, the shorter the domains, the smaller the aperture should be. Therefore the signals become extremely weak at the shorter domains. For this reason, MSR is thought to be inadequate as the readout of extremely small domains.
MAMMOS (Magnetic AMplifying Magneto-Optical System) readout can achieve both high resolution and large signals simultaneously [7]. It is therefore one of the most promising candidates for a next generation MO system. This paper describes MAMMOS readouts with magnetic field modulation (MFM-MAMMOS).

\section{MAMMOS Readouts}

The key layers in the disk structure for MAMMOS readouts are the readout layer, the recording layer and the gate layer. Figure 1 shows the principle of the MAMMOS readout. In a MAMMOS disk, a domain in the recording layer is copied to the readout layer through the gate layer, and the copied domain is expanded to a size that is larger than the diameter of the laser spot by using the external magnetic field. The gate layer switches the magnetic couplings between the readout and recording layer. In the readout process, a small recorded domain is selectively copied to the readout layer by the gate layer, then the domain is expanded in the readout layer by the external magnetic field. We can obtain a large signal by reproducing the expanded domain. After that, we can remove the domain in the readout layer by applying the external magnetic field in reverse. The signals are obtained continuously by repeating the process of expansion, readout and erasure.

The signals are large enough, and do not depend on the domain size because the reproduced domain is expanded

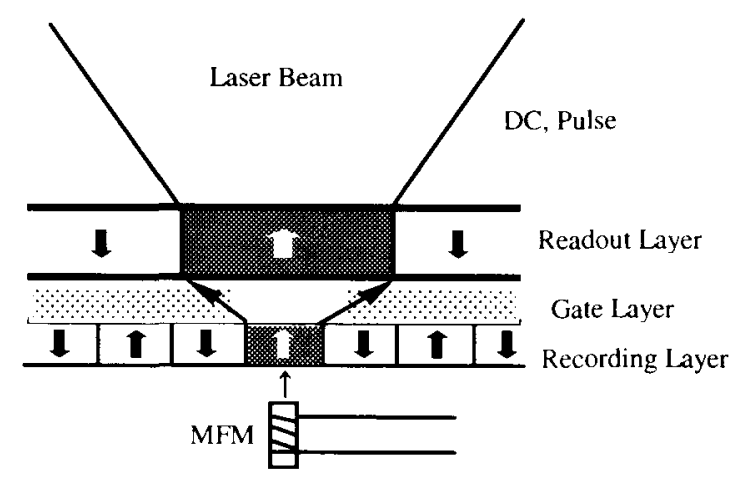

Fig. 1 Principle of MAMMOS. 
Table 1 Experimental conditions.

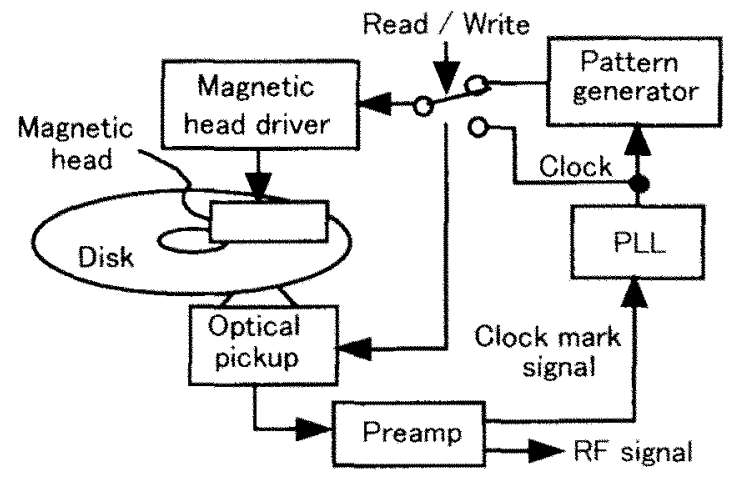

Fig. 2 Experimental setup for MFM-MAMMOS.

\begin{tabular}{ll}
\hline SiN & $70 \mathrm{~nm}$ \\
\hline TbFeCo & $200 \mathrm{~nm}$ \\
\hline AlTi & $10 \mathrm{~nm}$ \\
\hline SiN & $15 \mathrm{~nm}$ \\
\hline GdFeCo & $20 \mathrm{~nm}$ \\
\hline SiN & $70 \mathrm{~nm}$ \\
\hline \multicolumn{2}{l}{ PC Substrate } \\
\hline
\end{tabular}

Fig. 3 Structure of MAMMOS disk.

beyond the diameter of the readout laser spot. There are various MAMMOS classified by expansion methods such as, pulse/DC magnetic field or laser heating [7][8][9]. As mentioned above, MFM-MAMMOS is achieved by applying the external magnetic field for reading. Steady signals are easily obtained using a simple disk structure, although this method needs the external magnetic field for reading.

\section{Experimental Setup for MFM-MAMMOS}

In the MFM-MAMMOS system, the external magnetic fields should be synchronized with recorded domains. Figure 2 shows a schematic diagram of the recording/readout system for MFM-MAMMOS [10]. The system consists of an optical pickup, a magnetic head, a phase locked loop (PLL) circuit to generate a clock signal, and a pattern generator to generate a modulated signal for recording. The clock signal was synchronized with the embossed clock marks on the disk, and the recording/readout was performed using the clock signal. The external magnetic field, which was generated by the clock signal, was applied during readout for domain expansion.

The disk has embossed clock marks for the external clock system. The PLL circuit generates the clock signal by using the signal detected from the clock marks with the tangential push-pull method. The full disk structure is shown in Fig. 3. The disk consists of: PC substrate / SiN

\begin{tabular}{ll}
\hline Laser Wavelength & $635 \mathrm{~nm}$ \\
Lens N.A. & 0.55 \\
Track pitch & $0.6 \mu \mathrm{m}$ Land/Groove \\
Min. Mark Length & $0.04 \mu \mathrm{m}$ \\
Min. Space Length & $0.14 \mu \mathrm{m}$ \\
Disk Diameter & $120 \mathrm{~mm}$ \\
Disk thickness & $1.2 \mathrm{~mm}$ \\
Linear velocity & $0.6 \mathrm{~m} / \mathrm{sec}$ \\
MFM frequency & 6.0 to $12 \mathrm{MHz}$ \\
W/R strategy & MFM $/ \mathrm{MFM-MAMMOS}$ \\
Data Encoding & NRZI \\
Clocking & External by Fine Clock Mark
\end{tabular}

$70 \mathrm{~nm} / \mathrm{GdFeCo} 20 \mathrm{~nm} / \mathrm{SiN} 15 \mathrm{~nm} / \mathrm{AlT} 10 \mathrm{~nm} /$ TbFeCo $200 \mathrm{~nm} / \mathrm{SiN} 70 \mathrm{~nm} / \mathrm{UV}$ resin. The MAMMOS readout layer is $\mathrm{GdFeCo}$ and the recording layer is $\mathrm{TbFeCo}$.

The experimental conditions are listed in Tab. 1. The disk substrate was made of $\mathrm{PC}$, and the thickness of the substrate was $1.2 \mathrm{~mm}$. Lands/grooves with a track pitch of $0.6 \mu \mathrm{m}$ were formed. The write strategy was MFM recording. Carrier to noise ratio (CNR) was measured using an FFT analyzer, and bit error rate (BER) was measured by counting the missing and extra pulse of the readout signal from the packed domain.

\section{Read/Write Characteristics}

In a MAMMOS readout, the signal amplitude is just the same as that from a long domain. Figure 4 shows the CNR of MAMMOS, CAD-MSR and conventional disks. In both MSR and conventional disks, the CNR degrades with decreasing domain size. On the other hand, the CNR of the MAMMOS disk was more than $50 \mathrm{~dB}$, even at a $0.2 \mu \mathrm{m}$ packed domain. The MAMMOS disk showed high CNR

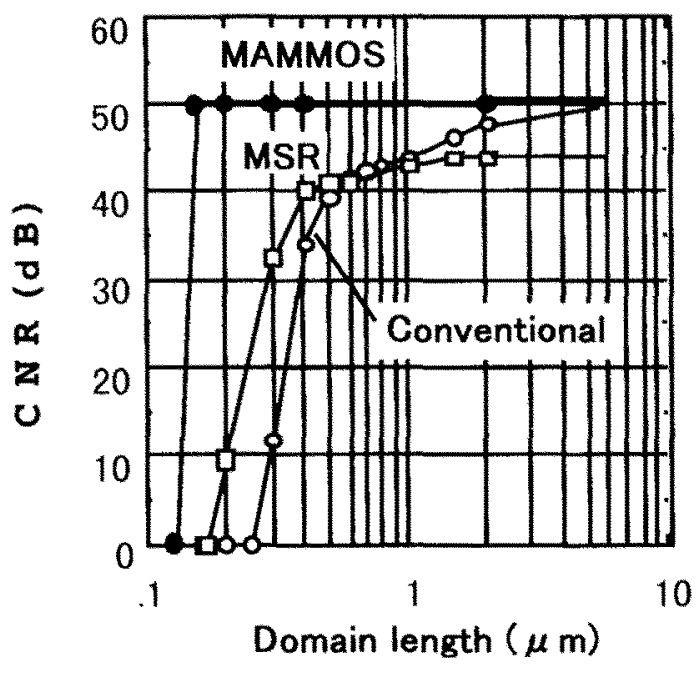

Fig. 4 Carrier to noise ratio (CNR) of MAMMOS. 


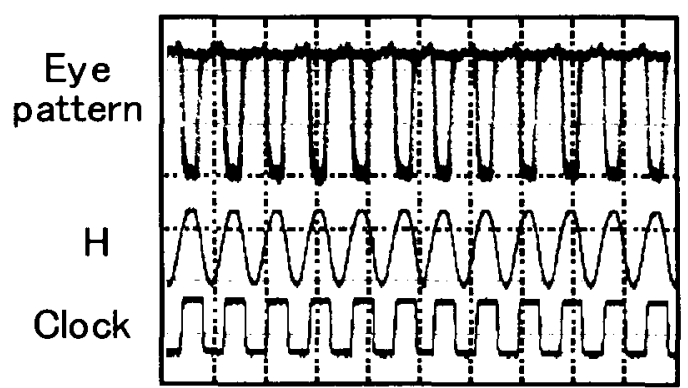

Fig. 5 Eye pattern of MAMMOS.

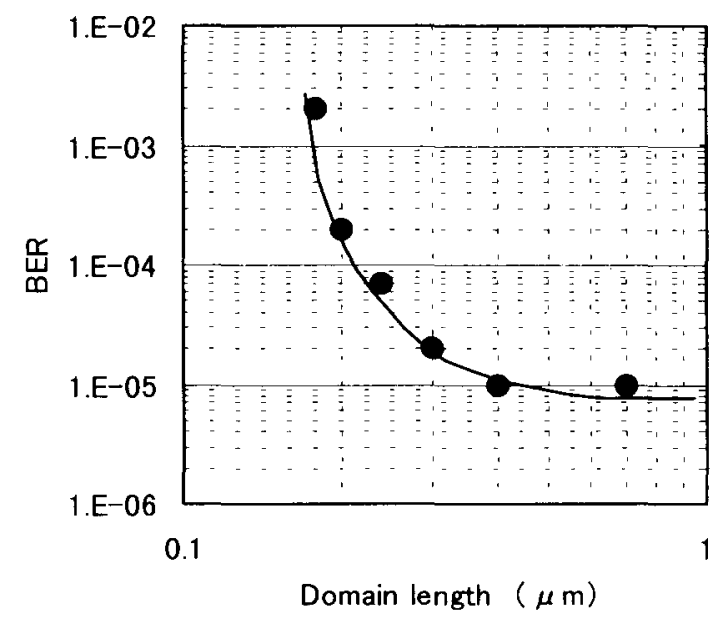

Fig. 6 Domain length dependence of bit error rate (BER).

not only at short domains, but also at long domains. The disk had a wide signal band with good SNR. However, the readout resolution depends on the aperture size for copying a domain. At a certain domain length, the signal suddenly decreased because the interval between each domain became smaller than the area in which the recorded domains were copied to the readout layer through the gate layer.

Figure 5 shows the eye pattern of the readout signal, applied magnetic field, and clock signal. The lower waveform is the clock signal generated from the clock marks that were prepared on the disk, the middle waveform is the applied magnetic field, and the top waveform is the eye pattern that was obtained by applying the magnetic field with a bit density of $0.2 \mu \mathrm{m} / \mathrm{bit}$. The shape of the eye pattern was very different from that of the conventional readout method. Since MAMMOS was able to reproduce a high quality readout signal, it is possible to discriminate the signal by sampling with the clock timing.

The domain length dependence of BER is shown in

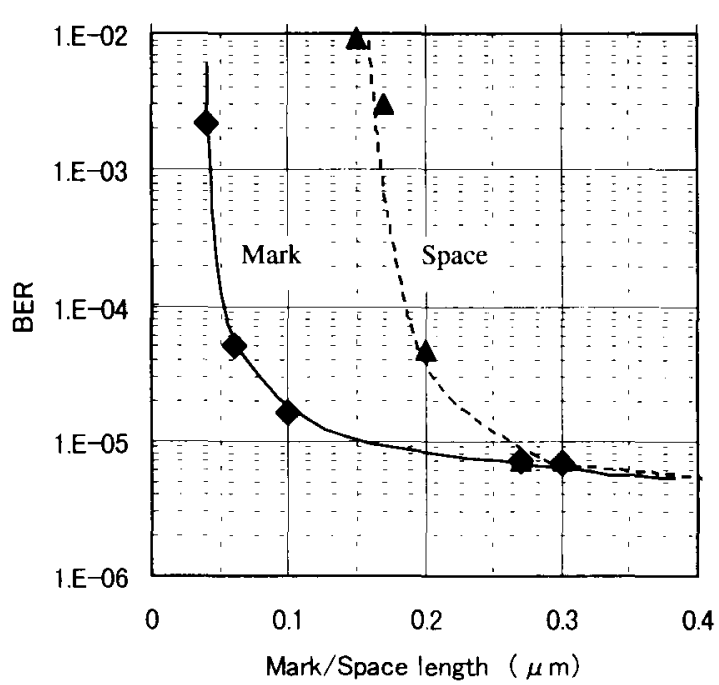

Fig. 7 Bit error rate (BER) of isolated marks and spaces.

(a) Mark length $0.06 \mu \mathrm{m}$

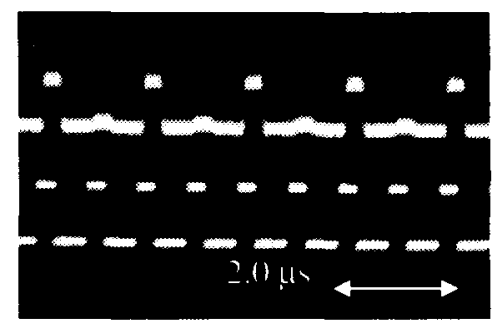

MAMMOS Signal

Ext. Clock

(b) Mark length $0.04 \mu \mathrm{m}$

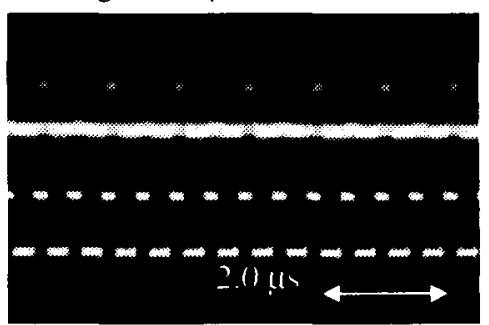

MAMMOS Signal

Ext. Clock

Fig. 8 MAMMOS readout signals.

Fig. 6, where read power and applied magnetic field were optimized individually. BER was in the $10^{-4}$ range even at a domain length of $0.2 \mu \mathrm{m}$. However, at a domain length of below $0.2 \mu \mathrm{m}$, the BER suddenly increased because the interval between each domain became smaller than the area where the recorded domains were copied to the readout layer.

To investigate the reason for the sudden increase of BER, we measured the BER of isolated marks and spaces separately. The results are shown in Fig. 7, where spaces were more than $0.6 \mu \mathrm{m}$ for isolated marks, and marks were 
more than $0.45 \mu \mathrm{m}$ for isolated spaces. The read power was $1.8 \mathrm{~mW}$ in both cases. Bit errors for spaces and marks are generated from the extra and missing pulse of the readout signal, respectively. The BER of spaces was almost the same as the BER in Fig. 6. On the other hand, the BER of marks was much lower than in Fig. 6. This means that the BER is determined by the spaces between marks. By setting the length of marks shorter than the length of spaces, the average domain length can be reduced.

Figure 8 shows the MAMMOS signal from isolated domains of (a) $0.06 \mu \mathrm{m}$ and (b) $0.04 \mu \mathrm{m}$. The signal was clearly reproduced from $0.06 \mu \mathrm{m}$ domains. Even at 0.04 $\mu \mathrm{m}$ domains, the signal amplitude was the same as the saturation signal level. The MAMMOS disk showed good characteristics for reproducing small domains.

\section{Conclusion}

We demonstrated that the MAMMOS readout was one of the most effective methods for high density recordings. The disk gave high performances in both large signal and high resolution. The CNR was more than $50 \mathrm{~dB}$ even at a domain length below $0.2 \mu \mathrm{m}$. We also obtained the MAMMOS signal from $0.04 \mu \mathrm{m}$ domains. The MAMMOS disk has a wider signal band than conventional disks. Combining the disk with signal processing techniques such as partial response maximum likelihood (PRML), will enable us to obtain media with larger capacity.

The capacity of optical disks has mainly been improved by shorter wavelength lasers. However, there is strong potential to dramatically improve capacity by using the magnetic domain expansion technique. The principle of this technique is based on the MSR disk and bubble memory. It is expected that a new method of domain expansion to be developed from these technologies.
Before long, creating a MAMMOS disk with a capacity of more than 100 Gbytes using a blue laser diode will no longer be a dream.

Acknowledgement The authors would like to thank to Mr. O. Suzuki, director of the Hypermedia Research Center, and Dr. K. Torazawa, for their continuous encouragement and helpful discussions.

\section{Rerefences}

[1] K. Aratani, A. Fukumoto, M. Ohta, M. Kaneko and K. Watanabe : Proc. SPIE, Optical Data Storage '91, Vol. 1499, p. 209 (1991)

[2] J. Hirokane and A. Takahashi : Jpn. J. Appl. Phys., Vol. 35, p. 5701 (1996)

[3] Y. Tanaka, S. Sumi, N. Matsubayashi, H. Sato, Y. Murakami, H. Awano, M. Matsuura, G. Fujita and T. Watanabe : Jpn. J. Appl. Phys., Vol. 37, p. 2150 (1998)

[4] T. Yamagami, S. Senshu, K. Seo, T. Watanabe and Y. Aoki : Proc. SPIE, Optical Data Storage '90, Vol. 1316, p. 271 (1990)

[5] H. Awano, H. Shirai, H. Watanabe, K. Shimazaki, M. Yoshihiro and N. Ohta : Proc. Magneto-Optical Recording Int. Symp. '97, Vol. 22 (Suppl. No. S2), p. 215 (1998)

[6] H. Awano, S. Ohnuki, H. Shirai, N. Ohta, A. Yamaguchi, S. Sumi and K. Torazawa : Appl. Phys. Lett., Vol. 69, No. 27, p. 4257 (1996)

[7] H. Awano, S. Ohnuki, H. Shirai, N.Ohta, A. Yamaguchi, S. Sumi and K. Torazawa : Proc. SPIE, Optical Data Storage '97, Vol. 3109, p. 83 (1997)

[8] A. Yamaguchi, Y. Suzuki, K. Tanase, S. Sumi and K. Torazawa : IEEE Trans. Magn., Vol. 33, No. 5, p. 3217 (1997)

[9] T. Shiratori, E. Fujii, Y. Miyaoka and Y. Hozumi : Proc. Magneto-Optical Recording Int. Symp. '97, Vol. 22 (Suppl. No. S2), p. 47 (1998)

[10] N. Takagi, A. Yamaguchi, S. Sumi, H. Awano, K. Shimazaki and N Ohta : Proc. SPIE, Optical Data Storage '98, Vol. 3401, p. 206(1998) 